\title{
The quest to improve sudden cardiac death prediction using sympathetic innervation scintigraphy: Chasing a mirage?
}

\author{
Firas J. Al Badarin, MD, ${ }^{\mathrm{a}, \mathrm{b}}$ and Poghni Peri-Okonny, MD MSc ${ }^{\mathrm{a}, \mathrm{b}}$ \\ a School of Medicine, University of Missouri-Kansas City, Kansas City, MO \\ b Department of Cardiovascular Research, Saint Luke's Mid America Heart Institute, Kansas City, \\ MO
}

Received Apr 19, 2019; accepted Apr 19, 2019

doi: 10.1007/s12350-019-01741-9

\begin{tabular}{|llll|}
\hline Abbreviations & $m$ IBG & Meta-iodobenzylguanidine \\
CHF & Congestive heart failure & SCD & Sudden cardiac death \\
ICD & Intracardiac defibrillator & & \\
LVEF & Left ventricular ejection fraction & & \\
\hline
\end{tabular}

\section{See related article, pp. 992-1001.}

Treatment options for patients with congestive heart failure (CHF) have evolved substantially over the last two decades to currently include various pharmaceutical and device-based therapies that improve clinical outcomes and quality of life in this population. For example, implantable cardiac defibrillators (ICDs) are now the cornerstone of primary prevention of sudden cardiac death (SCD) in patients with reduced left ventricular ejection fraction (LVEF), ${ }^{1}$ supported by significant improvement in overall survival in device recipients. $^{2}$

However, the current paradigm for selecting candidates for expensive interventions-such as ICD implantation-may need to be refined, especially as we continue to emphasize the need for individualized management decisions and to advocate for improving patients' outcomes without excessively increasing cost

Reprint requests: Firas J Al Badarin, MD, School of Medicine, University of Missouri-Kansas City, 4401 Wornall Rd, Kansas City, MO, 64111; falbadarin@yahoo.com

J Nucl Cardiol 2020;27:1002-4.

$1071-3581 / \$ 34.00$

Copyright (c) 2019 American Society of Nuclear Cardiology. to the society. Since LVEF does not adequately discriminate between patients who will or will not have a serious arrhythmic event after device implantation, ${ }^{3}$ there continues to be interest in identifying patients who, despite a reduced LVEF, may be less likely to derive benefit from ICD implantation. Therefore, several clinical, electrocardiographic, and imaging markers of increased susceptibility to arrhythmic events have been described. ${ }^{4}$ Assessment of cardiac sympathetic innervation with meta-iodobenzylguanidine ( $m \mathrm{IBG})$ scintigraphy has particularly emerged as a promising noninvasive tool for risk stratification in patients with $\mathrm{CHF}^{5,6}$

In this issue of the Journal, Ikeda-Yorifuji and colleagues $^{7}$ provide additional data to a space where more research is direly needed. The authors sought first to externally validate the ADMIRE-HF score, a previously described approach for risk stratification using cardiac $m$ IBG added to LVEF and systolic blood pressure. ${ }^{8}$ For this purpose, they applied the score in a cohort of 90 patients on stable guideline-directed medical therapy who had left ventricular systolic dysfunction (LVEF $<40 \%$ ) but did not have ICD at baseline. More than half of the patients had ischemic heart disease, and all underwent planar cardiac $m \mathrm{IBG}$ imaging at baseline and were followed prospectively for a median of 7.5 years. Patients with a high ADMIRE-HF score (>15) were significantly more likely to have serious arrhythmic events-defined as SCD, sustained VT, or 
Table 1. Prospective randomized clinical trials proposed to evaluate the utility of cardiac mIBG imaging in patients with congestive heart failure

Studies Study aims

Status

ADMIRE-ICD: international study to determine if AdreView heart function scan can be used to identify patients with mild or moderate heart failure (HF) that benefit from implanted medical device (NCT02656329)

MISTIC: $m$ IBG scintigraphy as a tool for selecting patients requiring implantable cardioverter defibrillator (NCT01 185756)

PET/CT With ${ }^{18} \mathrm{~F}$-FDG: does it optimize the ${ }^{123} \mathrm{I}-$ $m$ IBG imaging results in the search for discriminating factors for the implementation of an implantable defibrillator?

(NCT01258283)

Cardiac resynchronization and iodine metaiodobenzylguanidine (mIBG) imaging (NCT01522378)

To demonstrate the efficacy of cardiac mIBG imaging for appropriately guiding the decision of ICD implantation, in heart failure patients $(35 \% \geq \operatorname{LVEF} \geq 25 \%$ )

To assess if cardiac innervation in patients with heart failure can help better select candidates for an implantable cardioverter defibrillator

To determine if the number of discordant segments (mismatch) differs between patients with and without severe arrhythmia

To determine whether response to cardiac resynchronization therapy is associated with improvement in cardiac sympathetic innervation
Suspended

due to recruitment rate

Active; not recruiting

Terminated due to slow recruitment

Terminated

Data in the table abstracted from www.clinicaltrials.gov, last accessed on April 15th, 2019

appropriate device therapy-compared to those who had an intermediate $(4-15)$ or low $(<4)$ sores, with roughly 3- or 4-fold increased odds for having a serious arrhythmic event, respectively. In their second aim, the authors examined whether the presence of repolarization abnormalities on a resting electrocardiogram would be independently associated with future risk of SCD and other serious arrhythmic events, beyond the ADMIREHF score. Similarly, they report that patients with early repolarization changes had higher event rates compared to those without early repolarization changes, regardless of their ADMIRE-HF score.

The investigators are to be lauded for their efforts, and the strengths in their study need to be highlighted. First, both markers investigated by the authors-cardiac $m$ IBG and early repolarization-can be biologically linked to the process of arrhythmogenesis, and therefore their association with arrhythmic events is biologically plausible. ${ }^{9,10}$ Second, ascertainment of SCD was rigorous using a combination of electronic health records review and direct contact with patients, their families, or care providers. Lastly, the fact that none of the enrolled patients had an ICD at baseline allowed the prognostic utility of cardiac $m$ IBG imaging to be studied in this population without the modifying effect that ICD therapy has on the patients' survival trajectories. Notwithstanding these strengths, the study has several limitations that warrant further discussion. This cohort of 90 patients was recruited over a 5-year period, and the readers are not told a great deal about the number of patients who failed screening and were thus excluded. Such information will help the readers better understand how representative these patients are of the general heart failure population. Furthermore, the number of patients included, and subsequently the number of SCD events, were notably small. The evidently wide confidence intervals around the point estimates for the hazard ratios suggest that the point estimates reported are likely inflated due to high variance. In order to adequately adjust for the covariates chosen by the investigators without overfitting the model, four to five times the current number of events would be needed. Therefore, while it would be very intriguing to use the current data to provide external validation of the ADMIRE-HF score, these data fall short because of the limitations mentioned.

Following the release of the ADMIRE-HF (AdreView Myocardial Imaging for Risk Evaluation in Heart Failure) trial results, the FDA approved the use of AdreView $^{\circledR}$ (Iobenguane ${ }^{123} \mathrm{I}$ ) in patients with heart failure as a new indication for the purpose of refining cardiac risk stratification. ${ }^{11}$ Uptake of cardiac sympathetic innervation imaging, however, has been slow, in part due to uncertainty as to how this technology can be incorporated in the routine care of heart failure 
patients. ${ }^{12}$ Several prospective randomized trials have been initiated with the hope of providing evidence on how the results of cardiac $m$ IBG scintigraphy can inform management decisions in patient with CHF, particularly ICD implantation (Table 1). Unfortunately, most of these studies have been suspended due to slow recruitment, leaving the question unanswered as to how cardiac $m$ IBG scintigraphy can guide management decisions in this population.

In the current era of value-based care, emphasis on "high-quality" imaging will only continue to grow. ${ }^{13}$ For cardiac $m I B G$ scintigraphy to show its utility, there needs to be evidence that information provided by this imaging modality can alter management decisions and ultimately leads to improvement in patients' clinical outcomes. For this to be accomplished, it is essential to cultivate a strong partnership between key stakeholders-including nuclear cardiologists, heart failure specialists, outcomes researchers, and industry-to help design and conduct clinical studies needed to generate the needed evidence base. This will hopefully provide the foundation for broader and more patient-centered use of this imaging technology. Otherwise, we may just be chasing a mirage!

\section{Disclosures}

Drs. Al Badarin and Peri-Okonny are supported by a Grant from the National Heart, Lung, And Blood Institute of the National Institutes of Health (Award Number T32HL110837).

\section{References}

1. Nanthakumar K, Epstein AE, Kay GN, Plumb VJ, Lee DS. Prophylactic implantable cardioverter-defibrillator therapy in patients with left ventricular systolic dysfunction: A pooled analysis of 10 primary prevention trials. J Am Coll Cardiol 2004;44:2166-72.

2. Epstein AE, Dimarco JP, Ellenbogen KA, et al. ACC/AHA/HRS 2008 Guidelines for device-based therapy of cardiac rhythm abnormalities. Heart Rhythm 2008;5:e1-62.

3. Buxton AE. Should everyone with an ejection fraction less than or equal to $30 \%$ receive an implantable cardioverter-defibrillator? Not everyone with an ejection fraction $<$ or $=30 \%$ should receive an implantable cardioverter-defibrillator. Circulation 2005;111:2537-49; discussion 2537-49.

4. Goldberger JJ, Cain ME, Hohnloser SH, American Heart Association/American College of Cardiology Foundation/Heart Rhythm Society Scientific Statement on Noninvasive Risk Stratification Techniques for Identifying Patients at Risk for Sudden Cardiac Death, et al. A scientific statement from the American Heart Association Council on Clinical Cardiology Committee on Electrocardiography and Arrhythmias and Council on Epidemiology and Prevention. J Am Coll Cardiol 2008;52:1179-99.

5. Bax JJ, Kraft O, Buxton AE, et al. ${ }^{123} \mathrm{I}-m \mathrm{IBG}$ scintigraphy to predict inducibility of ventricular arrhythmias on cardiac electrophysiology testing: A prospective multicenter pilot study. Circ Cardiovasc Imaging 2008;1:131-40.

6. Nagahara D, Nakata T, Hashimoto A, et al. Predicting the need for an implantable cardioverter defibrillator using cardiac metaiodobenzylguanidine activity together with plasma natriuretic peptide concentration or left ventricular function. J Nucl Med 2008;49:225-33.

7. Ikeda-Yorifuji I, Yamada T, Tamaki S, et al. Prediction of sudden cardiac death in chronic heart failure patients with reduced ejection fraction by ADMIRE-HF risk score and early repolarization pattern. J Nucl Cardiol 2019. https://doi.org/10.1007/s12350-01901639-6.

8. Al Badarin FJ, Wimmer AP, Kennedy KF, Jacobson AF, Bateman TM. The utility of ADMIRE-HF risk score in predicting serious arrhythmic events in heart failure patients: Incremental prognostic benefit of cardiac ${ }^{123}$ I- $m$ IBG scintigraphy. J Nucl Cardiol 2014;21:756-62; quiz 753-5, 755-63.

9. Haïssaguerre M, Derval N, Sacher F, et al. Sudden Cardiac Arrest Associated with Early Repolarization. N Engl J Med 2008;358:2016-23.

10. Chen LS, Zhou S, Fishbein MC, Chen PS. New perspectives on the role of autonomic nervous system in the genesis of arrhythmias. $\mathrm{J}$ Cardiovasc Electrophysiol 2007;18:123-7.

11. Jacobson AF, Senior R, Cerqueira MD, et al. Myocardial iodine123 meta-iodobenzylguanidine imaging and cardiac events in heart failure. Results of the prospective ADMIRE-HF (AdreView Myocardial Imaging for Risk Evaluation in Heart Failure) study. J Am Coll Cardiol 2010;55:2212-21.

12. Scholte AJ. The future of cardiac 123-I $m$ IBG imaging. Eur J Nucl Med Mol Imaging 2016;43:2381-2.

13. Shaw LJ, Blankstein R, Jacobs JE, et al. Defining quality in cardiovascular imaging: A scientific statement from the American Heart Association. Circ Cardiovasc Imaging 2017. https://doi.org/ 10.1161/HCI.0000000000000017.

Publisher's Note Springer Nature remains neutral with regard to jurisdictional claims in published maps and institutional affiliations. 